\title{
On the Question of the Problems of Statistical Calculations of the Conducting Crystals Thermodynamic and Kinetic Properties
}

\author{
${ }^{1}$ Lviv Polytechnic National University, Lviv, Ukraine, e-mail: jabudjak@ukr.net \\ ${ }^{2}$ Krakow Polytechnic, Krakow, Poland, e-mail: tadeuszwaclawski00@gmail.com
}

\begin{abstract}
This paper presents an elementary model of a crystal and its thermodynamic equilibrium state. It was shown that the thermodynamic characteristics of the crystal at this state are described by the Gibbs grand thermodynamic potential. If the crystal is removed away from the equilibrium state, then in this state it will be described by the set of kinetic properties, and these properties are statistically calculated with the use of the non-equilibrium Gibbs grand thermodynamic potential. Crystals' thermodynamic and kinetic properties have analytical dependence on the current carriers dispersion law and chemical potential of these carriers. In this work, it was shown that the determination of the dispersion law and chemical potential - these are complicated problems of statistical and kinetic theories of crystals' properties.
\end{abstract}

Keywords: Gibbs potential, dispersion law, chemical potential, drift force.

Article acted received 04.11.2019; accepted for publication 15.12.2019.

\section{An elementary model of a crystal. The thermal and kinetic properties of crystals}

Crystals are composed of the structural particles and they are located within a crystal volume. The structural particles create the crystal lattice. This lattice has a symmetry. Points of the location of the structural particles are called the lattice sites. The space between these particles is called the interstitial site. As the crystal structural particles there are atoms, ions or molecules. The interaction between these particles holds them in the lattice sites.

In a crystal can exist the free charge carriers and when the crystal is in the thermodynamic equilibrium state, these carriers are moving chaotically in the crystal interstitial site. Experiments show that electrons with the charge $e$ or positive holes with the charge $-e$ can be the free charge carriers in crystals. A collection of the charge carriers in the crystal is called the electron or hole gas, or called as the gas of the charge carriers.

In thermodynamic equilibrium, there is not any action of forced fields on the crystal, and its temperature remains constant and the same value in all its points. In all crystal states its structural particles hamonically vibrate around their nodes, the directions of these vibrations are varying chaotically, and the charge carriers gas is moving chaotically in the interstitial sites of the crystal lattice. It is agreed to think that the system of structural particles and the system of charge carriers only weakly interact with each other.

Thus, the crystal as a thermodynamic system, is composed of two thermodynamic subsystems of particles. One subsystem - this is the gas of charge carriers, this gas is considered to be an ideal. The other this a set of structure particles, which harmonically and chaotically vibrate around nodes of crystal lattice.

The thermal properties of conducting crystals are determined by the concentration of free charge carriers and the character of their motion in the crystal interstitial site.

In the thermodynamic equilibrium state, the free charge carriers are moving in a chaotic way, their average energy is conserved, and the entropy of the system of these carriers obtains its maximum value. This is an equilibrium gas of the charge carriers. As it was shown in the works [1-5], this gas is described by the Gibbs grand canonical thermodynamic potential: 


$$
\Omega=-2 V \int_{0}^{\infty} \frac{G(\varepsilon)}{\left(\exp \left(\frac{\varepsilon-\mu}{k T}\right)+1\right)} d \varepsilon=-2 V \int_{0}^{\infty} G(\varepsilon) f_{0}(\varepsilon, \mu) d \varepsilon
$$

In this formula, $V$ is the crystal volume, $f_{0}(\varepsilon, \mu)=$ $\left(\exp \left(\frac{\varepsilon-\mu}{k T}\right)+1\right)^{-1}$ is the Fermi-Dirac function, $G(\varepsilon)=$ $\int_{0}^{\varepsilon} g(\varepsilon) d \varepsilon, g(\varepsilon)$ is the density of the energy states (DOS) of charge carriers lying in allowed band.

Furthermore, with the use of the potential (1) and methods of statistical thermodynamics, it can be shown that the thermodynamic functions of the charge carriers' gas are given by the following general formulas:

$$
\begin{gathered}
U=\Omega-\left(\frac{d \Omega}{d \mu}\right)_{T} \mu-\left(\frac{d \Omega}{d T}\right)_{\mu} T, \\
H=-\left(\frac{d \Omega}{d \mu}\right)_{T} \mu-\left(\frac{d \Omega}{d T}\right)_{\mu} T \\
F=\Omega-\left(\frac{d \Omega}{d \mu}\right)_{T} \mu
\end{gathered}
$$

$$
\begin{gathered}
G=-\left(\frac{d \Omega}{d \mu}\right)_{T} \mu=N \mu \\
\Omega=-2 V \int_{0}^{\infty} G(\varepsilon) f_{0}(\varepsilon, \mu) d \varepsilon \\
S=-\left(\frac{d \Omega}{d T}\right)_{\mu}=\frac{U-F}{T}, \\
C_{V}=\left(\frac{d U}{d T}\right)_{V} \\
P V=-\Omega, \\
N=-\left(\frac{d \Omega}{d \mu}\right)_{T}
\end{gathered}
$$

and thus, we have:

$$
n\left(\mu^{\bullet} T\right)=\int_{0}^{\infty} g(\varepsilon) f_{0}(\varepsilon, \mu) d \varepsilon=\int_{0}^{\infty} G(\varepsilon)\left(-\frac{d f_{0}(\varepsilon, \mu)}{d \varepsilon}\right) d \varepsilon=\int_{0}^{\infty} G(x \cdot k T)\left(-\frac{d f_{0}\left(x, \mu^{\bullet}\right)}{d x}\right) d x
$$

The analysis of the above formulas shows us that the all set of thermal properties of the charge carriers' gas in a crystal is fully described by the potential (1) and this potential depends on the energy dispersion relation via $G(\varepsilon)$ and the reduced chemical potential $\mu^{\circ}$.

Thermal properties of the crystal lattice are described by the well-known Debye potential $[4,5]$ :

$$
\Omega_{D}=\Omega_{D}(N, \theta, T) .
$$

In this formula, $N$ is the general amount of the crystal structural particles, $\theta$ is the parameter of the crystal lattice named the Debye temperature.

The calculations of these thermal properties of the crystal lattice, with the use the potential (2), are given in the cited literature.

The general thermal properties of a crystal are additive, and their values for an entire crystal are calculated by summing the values of thermal properties of the crystal lattice and the thermal properties of the charge carriers' gas in this crystal.

Under some drift perturbations in a crystal, that is, the electric field $\overleftarrow{E}$, the temperature gradient $\nabla_{\bar{r}} T$ (these perturbations may exist simultaneously in a crystal), the charge carriers' gas will go out from equilibrium and turn into the non-equilibrium particles' ensemble. In this case, the drift force $\vec{F}_{d}$ will be act on every particle of charge $z e[4,5]$ :

$$
\overleftarrow{F_{d}}=z e \overleftarrow{E_{d}}, \quad \overleftarrow{E_{d}}=\overleftarrow{E}-\left(\frac{k}{z e}\right)\left(\frac{\varepsilon-\mu}{k T}\right) \nabla_{\dot{r}} T
$$

where $e$ is the electronic charge, $z= \pm 1$ is the sign of charge, $\varepsilon$ is the average energy of a charge carrier, $k$ is the Boltzmann's constant, $T$ is the crystal temperature.

As a result of the action of the drift force $\overleftarrow{F_{d}}$, all current carriers start to move in a rectilinear direction. Their drift velocity $\vec{v}_{d}$ depends on the force $\vec{F}_{d}$ and the crystal properties.

The presence of this drift velocity $\vec{v}_{d}$ forms a particle flux in the crystal. There is electric charge and heat (energy) transport in this crystal. Thus, under the drift fields in the crystal, the set of charge carriers turns into the non-equilibrium grand canonical ensemble with the varying amount of the particle. This grand canonical ensemble, as it was shown in work [1], when the spin degeneracy was taking into consideration, is defined by the Gibbs grand canonical thermodynamic potential:

$$
\Omega=-2 V \int_{0}^{\infty} \frac{G(\varepsilon)}{\left.\exp \left(\frac{\varepsilon-\mu-\Delta \mu}{k T}\right)+1\right)} d \varepsilon=-2 V \int_{0}^{\infty} G(\varepsilon) f_{0}\left(\varepsilon, \mu, \Delta \mu_{p}\right) d \varepsilon
$$

In this formula , $\vec{p}$ is the charge carrier wavevector, $\varepsilon=\varepsilon_{\vec{p}}$ is the charge carriers' energy dispersion relation, $\Delta \mu(\vec{p})$ is the change in the one particle chemical potential by the action of these perturbations (these will remove the crystal away from the equilibrium state). When the perturbations are absent, $\Delta \mu(\vec{p})=0$. 
The value $\Delta \mu(\vec{p})$ was calculated in the work[1], where it was shown that $\Delta \mu(\vec{p})$ is an odd function of the vector $\vec{p}$, and in an isotropic crystal under action of the magnetic induction vector $\vec{B}$, this value is given as:

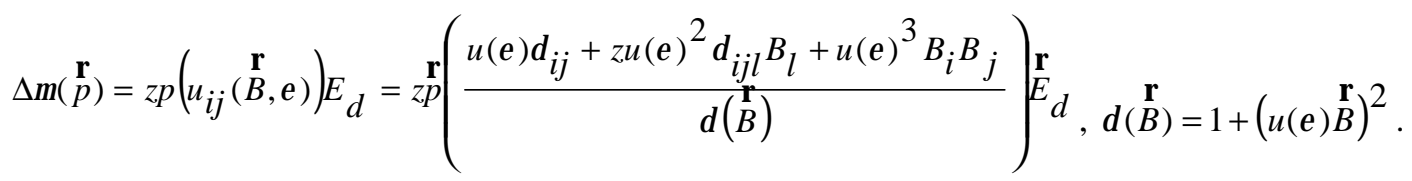

In this formula, $\delta_{i j}$ and $\delta_{i j l}$ are the well-known Kronecker delta and Levi-Civita symbols, and $u(\varepsilon)$ is the well-known scattering function and it describes the effect of scattering processes by crystal lattice defects on the crystal kinetic properties. crystal is described by the Gibbs's grand canonical thermodynamic potential (4)) the electric charge and heat transport processes exist, and they are described by the following generalized electrical and heat conduction equations:

\section{Analysis of statistical calculations of the crystals' thermal and kinetic properties}

In the cited works, it was shown that in a crystal (this

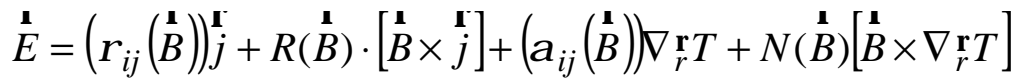

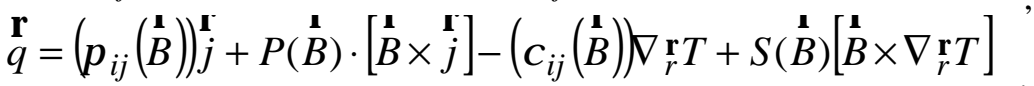

In equations (6), (7), the symmetric tensors $\left(\rho_{i j}(\vec{B})\right)$, $\left(\alpha_{i j}(\vec{B})\right),\left(\pi_{i j}(\vec{B})\right),\left(\chi_{i j}(\vec{B})\right)-$ these are respectively, the material tensors of the resistivity, Seebeck effect, Peltier effect and thermal conductivity of the crystal. They are even function of the magnetic induction vector $\vec{B}$.

The coefficients $R(\vec{B})$ and $P(\vec{B})$ - these are the coefficients of transverse galvanomagnetic Hall and Ettingshausen effects, and the coefficients $N(\vec{B})$ and $S(\vec{B})$ are the coefficient of transverse thermomagnetic Nernst-Ettigshausen and Righi-Leduc effects. In the isotropic crystals they are even and scalar function of the magnetic induction vector $\vec{B}$, that is: $R(\vec{B})=R(\overrightarrow{=B})$, $P(\vec{B})=P(\overrightarrow{-B}), N(\vec{B})=N(-\vec{B}), S(\vec{B})=S(-\vec{B})$.

The analysis of equations (6), (7) shows that in the presence of a magnetic field, an isotropic crystal becomes anisotropic, and the relativity simple processes of the electric and heat conduction will become more complicated. In this case, the additional transverse galvanomagnetic and thermomagnetic effects there occur.

The galvanomagnetic effects are produced by the action of a magnetic field on the omic part of the electrical current, and the thermomagnetic effect - by the action of this field on the thermal part of the current, accordingly to the generalized equations of the electric conduction (6).

The kinetic tensors and coefficients in equations (6), (7) describe the nature of the important material properties of conducting medium and they are of pragmatic significance for problems of the crystal properties prediction during synthesis of the crystals with prescibed properties. In the presence of a weak magnetic field in the crystal (when condition $(u(\varepsilon) \overleftarrow{B})^{2} \ll 1$ holds), or when the magnetic field is absent, all four kinetic tensors turn into scalars, and therefore all important kinetic properties of an isotropic crystal are described by the following set of scalars: $\rho\left(\mu^{\bullet}, T\right)$, $\alpha\left(\mu^{\bullet}, T\right), \pi\left(\mu^{\bullet}, T\right), \chi\left(\mu^{\bullet}, T\right), R\left(\mu^{\bullet}, T\right), N\left(\mu^{\bullet}, T\right), P\left(\mu^{\bullet}, T\right)$, $S\left(\mu^{\bullet}, T\right)$ and the concentration of charge carriers (current carriers) $n=n\left(\mu^{\bullet}, T\right)$.

All these kinetic coefficients and $n=n\left(\mu^{\bullet}, T\right)$ for macroscopic crystals, as it was shown in the works [1-5], can be calculated with the use of the following algorithmic functional:

$$
J\left(i, j, \mu^{\bullet}, T\right)=\int_{0}^{\infty} x^{i} u(x, T)^{j} G(x, T)\left(-\frac{d f_{0}\left(x, \mu^{\bullet}\right)}{d x}\right) d x
$$

With the use of this functional, in the presence of a weak magnetic field, or when the magnetic field is absent, the full set of kinetic properties of isotropic crystals will be described by the following formulas:

$$
\begin{gathered}
\rho\left(\mu^{\bullet}, T\right)=\frac{1}{\text { en }} \frac{J\left(0,0, \mu^{\bullet}, T\right)}{J\left(0,1, \mu^{\bullet}, T\right)}, \\
R\left(\mu^{\bullet}, T\right)=\frac{1}{z e n} \frac{J\left(0,0, \mu^{\bullet}, T\right) J\left(0,2, \mu^{\bullet}, T\right)}{J\left(0,1, \mu^{\bullet}, T\right)^{2}}, \\
\alpha\left(\mu^{\bullet}, T\right)=\frac{k}{z e}\left(\frac{J\left(1,1, \mu^{\bullet}, T\right)}{J\left(0,1, \mu^{\bullet}, T\right)}-\mu^{\bullet}\right), \\
N\left(\mu^{\bullet}, T\right)=\frac{k}{e} U_{H}\left(\mu^{\bullet}, T\right)\left(\frac{J\left(1,1, \mu^{\bullet}, T\right)}{J\left(0,1, \mu^{\bullet}, T\right)}-\frac{J\left(1,2, \mu^{\bullet}, T\right)}{J\left(0,2, \mu^{\bullet}, T\right)}\right),
\end{gathered}
$$




$$
\begin{aligned}
& \pi\left(\mu^{\bullet}, T\right)=T \alpha\left(\mu^{\bullet}, T\right), \\
& P\left(\mu^{\bullet}, T\right)=T N\left(\mu^{\bullet}, T\right), \\
& \chi\left(\mu^{\bullet}, T\right)=\left(\frac{k}{e}\right)^{2} \frac{T}{\rho\left(\mu^{\bullet}, T\right)}\left(\frac{J\left(2,1, \mu^{\bullet}, T\right)}{J\left(0,1, \mu^{\bullet}, T\right)}-\left(\frac{J\left(1,1, \mu^{\bullet}, T\right)}{J\left(0,1, \mu^{\bullet}, T\right)}\right)^{2}\right), \\
& U_{H}\left(\mu^{\bullet}, T\right)=\frac{J\left(0,2, \mu^{*}, T\right)}{J\left(0,1, \mu^{*}, T\right)}, U_{D}\left(\mu^{\bullet}, T\right)=\frac{J\left(0,1, \mu^{*}, T\right)}{J\left(0,0, \mu^{*}, T\right)}, \\
& n\left(\mu^{\bullet}, T\right)=J\left(0,0, \mu^{\bullet}, T\right) .
\end{aligned}
$$

The above formulas show that an anisotropic crystal (that its anisotropy was obtained by the action of a magnetic field) turns into an isotropic crystal, when the magnetic induction vector $\vec{B}$ fulfils the condtition $(u(\varepsilon) \overleftarrow{B})^{2} \ll 1$, or $B_{3}=0$.

The Gibbs potential (1) and functional (8) depend on the energy dispersion relation $\varepsilon(\overleftarrow{p)}$ (via $G(\varepsilon)$ ) and the chemical potential $\mu$ ( via the Fermi_Dirac function $\left.f_{0}(\varepsilon, \mu)=\left(\exp \left(\frac{\varepsilon-\mu}{k T}\right)+1\right)^{-1}\right)$. Therefore, the crystal thermal and kinetic properties have the same dependences on $\varepsilon(\overleftarrow{p})$ and $\mu$.

The energy dispersion relation is called the analytical formula $\varepsilon(\overleftarrow{p)}$, which describes the dependence of the crystal charge carrier energy $\varepsilon$ on this carrier quasimomentum $\grave{p}$.

The energy dispersion relation $\varepsilon(\overleftarrow{p)}-$ this is a difficult and an essential quantum-mechanical problem. For isotropic crystals, the quantum-mechanical calculations give basis for the following general isotropic dispersion relation:

$$
\frac{p_{x}^{2}+p_{y}^{2}+p_{x}^{2}}{2 m m_{n}}=\frac{p^{2}}{2 m m_{n}}=E(\varepsilon) .
$$

In this formula, $E(\varepsilon)$ is the quantum mechanical energy function of the first-order with respect to the parameter $k T, m$ is the electronic mass, $m_{n}$ is the charge carrier reduced effective mass in a crystal.

A function $E(\varepsilon)$ is called homogeneous function of the first-order if for all values of parameter $k T$, the condition $E(k T \varepsilon)=k T E(\varepsilon)$ holds.

The above analysis of the statististical calculations of the crystals thermal and kinetic properties shows that all these properties analytically depend on the dispersion relation (9). and all calculated parameters, in the algorithmic calculations, depend on the dispersion relation (9).

There are the following parameters: $g(\varepsilon)$ is the current carriers density of states (DOS) lying in allowed bands of crystals, $G(\varepsilon)=\int_{0}^{\varepsilon} g(\varepsilon) d \varepsilon, u(\varepsilon, T)$ is the dimensional scattering function which describes the action of the current carriers' scattering processes (in the defects of crystal lattice) on the crystals kinetic properties.

For the dispersion relation (9), these quantities take the forms:

$$
\begin{gathered}
G(\varepsilon)=\frac{8}{3 \sqrt{\pi}}\left(\frac{2 \pi m m_{n}}{h^{2}}\right)^{3 / 2} E^{3 / 2}(\varepsilon), \\
g(\varepsilon)=\frac{d G(\varepsilon)}{d \varepsilon}=\frac{4 \pi}{\sqrt{\pi}}\left(\frac{2 \pi m m_{n}}{h^{2}}\right)^{3 / 2} E^{1 / 2}(\varepsilon) \frac{d E}{d \varepsilon}, \\
G(x, T)=N_{C}(T) E^{3 / 2}(x), g(x, T) \\
=\frac{3}{2} \frac{1}{k T} N_{C}(T) E^{1 / 2}(x) \frac{d E}{d x}, \\
N_{C}(T)=\frac{8}{3 \sqrt{\pi}}\left(\frac{2 \pi m m_{n}}{h^{2}}\right)^{3 / 2}, \\
u(\varepsilon, T)=\mu^{(r)}(T) p^{(2 r-3)}\left(\frac{d \varepsilon}{d p}\right)^{2}=u^{(r)}(T) p^{(2 r-3)}\left(\frac{d p}{d \varepsilon}\right)^{-2}, \\
u(x, T)=U(r, T) \frac{E^{(r-1 / 2)}(x)}{\left(\frac{d E(x)}{d x}\right)^{2}} .
\end{gathered}
$$

In the last formula, $U(r, T)$ is the temperature function, it has a dimension of the mobility and it is described by the following formula:

$$
U(r, T)=\left[\frac{1}{T}\left(U_{A} \delta(0, r)+U_{O} \delta(1, r)\right)+U_{I} \delta(2, r)\right]\left(m_{n}\right)^{(r-5 / 2)} T^{(r-1 / 2)},
$$

where $U_{A}, U_{O}, U_{I}$ are the dimensional crystal constants, which depend on the nature of the crystal and the scattering mode of current carriers in the crystal lattice, $\delta(m, n)$ is the known Kronecker symbol, it has the following values: $\delta(m, n)=1$ for $m=n, \delta(m, n)=0$ for $m \neq n, r$ is the scattering parameter and it has the following values: $r=0$ for the scattering by the acoustic phonons in the crystal lattice, $r=1$ for the scattering by the optical phonons of the crystal lattice, $r=2$ for the scattering by the charged impurities (ions) of the crystal lattice.

For the dispersion relation (9), the algorithmic functional $J\left(i, j, \mu^{\bullet}, T\right)$ takes the form:

$$
\begin{aligned}
& J\left(i, j, \mu^{\bullet}, T\right)=\int_{0}^{\infty}\left(\frac{\varepsilon}{k T}\right)^{i} u(\varepsilon)^{j} G(\varepsilon)\left(-\frac{d f_{0}}{d \varepsilon}\right) d \varepsilon=U(r, T)^{j} N_{C}(T) \int_{0}^{\infty} x^{i} \frac{E(x)^{(r j-j / 2+3 / 2)}}{\left(\frac{d E(x)}{d x}\right)^{2 j}}\left(-\frac{d f_{0}}{d x}\right) d x= \\
& =U(r, T)^{j} N_{C}(T) I\left(i, j, \mu^{\bullet}, T\right),
\end{aligned}
$$

where the dimensionless integral $I\left(i, j, \mu^{\bullet}, T\right)$ is given as: 


$$
I\left(i, j, \mu^{\bullet}, \beta(T)\right)=\int_{0}^{\infty} x^{i} \frac{E(x)^{(r j-j / 2+3 / 2)}}{\left(\frac{d E(x)}{d x}\right)^{2 j}}\left(-\frac{d f_{0}}{d x}\right) d x
$$

The algorithmic formulas for the thermal and kinetic properties of crystals have an analytically dependence on the dispersion relation via the function $E(x)$ and on the reduced chemical potential $\mu^{\bullet}=\mu / k T$ via the FermiDirac function $f_{0}\left(x, \mu^{\bullet}\right)=\left(\exp \left(x-\mu^{\bullet}\right)+1\right)^{-1}$. In these relationship, the quantity $\mu$ denotes the charge carrier's free energy in a crystal (electron's or hole's energy), and $\mu^{\bullet}=\mu / k T$ is the reduced free energy of this charge carrier, which is called the reduced chemical potential.

In statistical physics it was shown that $\mu^{*}$ is a root of the known neutrality equation for the fabricated crystals. Thus, theoretical calculations of the chemical potential $\mu$ are associated with algebraic methods of finding solutions of the neutrality equations for crystals.

Consider n-type semiconductor crystal which is doped with both type of impurities, donors and acceptors. The concentrations of donors is $N_{D}$, and the concentrations of acceptors is $N_{A}$. The respective activation energies of them are $E_{D}, E_{A}$. As it was shown in the cited works $[4,5]$, there is the impurity screening by current carriers in such semiconductors, and the neutrality equation has the following form:

$$
n\left(\mu^{*}, T\right)=\frac{N_{D}}{1+2 \Phi(y-2) \cdot \exp \left(E_{D}^{*} \cdot F(x, y)+\mu^{*}\right)}-N_{A} .
$$

In this equation, $E_{D}^{\cdot}=E_{D} / k T, E_{A}^{\cdot}=E_{A} / k T$ there are the reduced activation energies of donors and acceptors,

$n\left(\mu^{\bullet}, T\right)=J\left(0,0, \mu^{\bullet}, T\right)=\int_{0}^{\infty} G(x, T)\left(-\frac{\partial f_{0}\left(x, \mu^{\bullet}\right)}{\partial x}\right) d x$ is the electron gas concentration in a crystal.

In consequence of this shielding, the donors' activation energy $E_{D}$ depends on the electron gas concentration and temperature, and this energy is given by the following general formula:

$$
E_{D}(T)=E_{D} F(x, y) \text {. }
$$

In this formula, $F(x, y)$ is the shielding function and it takes the form:

$$
F(x, y)=\left[2 \frac{(\operatorname{Re}(x)-1)^{3}}{y x^{2}}-\frac{(R e(x)-1)^{2}+\operatorname{Im}(x)^{2}}{y^{2}}\right]
$$

where $y=\frac{2 r_{0}}{a^{\bullet}}=\frac{1}{a_{0} e} \sqrt{\frac{k T}{\pi D}}\left(m_{n}\right)\left(\frac{d n\left(\mu^{\bullet}, T\right)}{d \mu^{\bullet}}\right)^{-1 / 2}$ is the shielding parameter, $r_{0}=$ $r_{0}\left(\mu^{\bullet}, T\right) 0 \sqrt{\frac{k T D}{4 \pi e^{2}}}\left(\frac{d n\left(\mu^{\bullet}, T\right)}{d \mu^{\bullet}}\right)^{-1 / 2}$ is the shielding radius. In these formulas, $a_{0}$ is the radius of a hydrogen atom, $D$ is the dielectric constant, $m$ is the free electron mass, $m_{n}$ is the reduced effective mass of a current carrier in a crystal, $\operatorname{Re}(x)$ and $\operatorname{Im}(x)$ are, respectively, the real and imaginary parts of a root of the following cubic equation:

$$
x^{3}-y x^{2}-y x+2 y=0 .
$$

This equation has tree roots. Under the condition of the calculation problem, which brings to formula (14), there is only one root having maximal positive value of $\operatorname{Re}(x)$.

The shielding function $F(x, y)$ has the following properties: $F(x, y)<0$ for $y<2, F(x, y)=0$ for $y=$ $2, F(x, y) \rightarrow 1$ for $y \gg 2$.

The Heaviside function $\Phi(y-2)$ in equation (13) has the following values: $\Phi(y-2)=1$ for $y \geq 2$ and $\Phi(y-2)=0$ for $y<2$.

From the formula for shielding parametr we have the following equation:

$$
\frac{d n}{d \mu^{\bullet}}=n \frac{d(\ln (n))}{d \mu^{\bullet}}=\frac{k T}{y^{2} \pi e^{2} a_{0}^{2} D}\left(m_{n}\right)^{2}=\frac{6.8 \cdot 10^{18}}{y^{2}}\left(m_{n}\right)^{2} \frac{T}{D}\left[\frac{\mathrm{cm}^{-3}}{K}\right] .
$$

For $y<2$, equation (13) has the following form:

$$
n\left(\mu^{\bullet}, T\right)=\int_{0}^{\infty} G(x)\left(-\frac{\partial f_{0}\left(x, \mu^{\bullet}\right)}{\partial x}\right) d x=N_{D}-N_{A} .
$$

It should be noted that when $y<2$, then by formula (15) the above equation is fulfilled the case of crystals with the high concentration of degenerate current carriers. In this connection, the integral in this equation can be calculated reasonable well with the Sommerfeld expansion and the equation obtains the following form

$$
n\left(\mu^{\cdot}, T\right)=G\left(\mu_{0}\right)+\left(\mu-\mu_{0}\right) g\left(\mu_{0}\right)+\frac{\pi^{2}}{6}(k T)^{2} \frac{d g\left(\mu_{0}\right)}{d \mu_{0}}=N_{D}-N_{A} .
$$


In this equation, $\mu_{0}$ is called the Fermi level. In its mathematical and physical meaning this equation may be divided into the following two equations:

$$
\begin{gathered}
G\left(\mu_{0}\right)=N_{D}-N_{A}=n\left(\mu_{0}\right), \\
\left(\mu-\mu_{0}\right) g\left(\mu_{0}\right)+\frac{\pi^{2}}{6}(k T)^{2} \frac{d g\left(\mu_{0}\right)}{d \mu_{0}}=0 .
\end{gathered}
$$

A root of this equation has the form

thus

$$
\mu=\mu_{0}\left(1-\frac{\pi^{2}}{6} \cdot \frac{(k T)^{2}}{\mu_{0} g\left(\mu_{0}\right)} \cdot \frac{d g\left(\mu_{0}\right)}{d \mu_{0}}\right),
$$

$$
\mu^{\cdot}=\mu_{0}^{\cdot}\left(1-\frac{\pi^{2}}{6} \cdot \frac{1}{\mu_{0}^{\bullet} g\left(\mu_{0}^{\bullet}\right)} \cdot \frac{d g\left(\mu_{0}^{\bullet}\right)}{d \mu_{0}^{\bullet}}\right) \text {, }
$$

here $\mu^{\bullet}=\frac{\mu}{k T}, \mu_{0}^{\bullet}=\frac{\mu_{0}}{k T}$ are, respectively, the reduced chemical potential and reduced Fermi level.

Finally, we have that the general solution of the neutrality equation (13), when $y<2$ and $\frac{d n}{d \mu^{-}}=$ $\frac{6.8 \cdot 10^{18}}{y^{2}}\left(m_{N}\right)^{2} \frac{T}{D}$, will be given by the following two equations:

$$
\begin{gathered}
G\left(\mu_{0}\right)=N_{D}-N_{A}=n\left(\mu_{0}\right) \\
\mu=\mu_{0}\left(1-\frac{\pi^{2}}{6} \cdot \frac{(k T)^{2}}{\mu_{0} g\left(\mu_{0}\right)} \cdot \frac{d g\left(\mu_{0}\right)}{d \mu_{0}}\right) .
\end{gathered}
$$

These equations show us that under these condition, heavily doped crystals have signs of metals. In metals, the current carriers concentration (16) does not depend on temperature, and the chemical potential decreases with a fall in temperature.

Under a condition that $y \gg 2$, the shielding function $F(x, y) \rightarrow 1$, thus equation (13) turns into the following equation:

$$
\begin{aligned}
& n\left(\mu^{\bullet}, T\right)=\int_{0}^{\infty} G(x)\left(-\frac{d f_{0}\left(x, \mu^{\bullet}\right)}{d x}\right) d x= \\
& -\frac{N_{D}}{1+2 \exp \left(E_{D}^{*}+\mu^{*}\right)}-N_{A}
\end{aligned}
$$

However, if a condition $y \gg 2$ holds, then from formula (15) it follows that the electron gas concentration is low and there is a non-degenerate gas in the crystal.

Then, we have:

$$
n\left(\mu^{\bullet}, T\right)=\int_{0}^{\infty} G(x)\left(-\frac{\partial f_{0}\left(x, \mu^{\bullet}\right)}{\partial x}\right) d x \cong
$$

$n\left(\mu^{\bullet}, T\right)=\int_{0}^{\infty} G(x) \cdot e^{-x} d x \cdot e^{\mu^{\bullet}}=Z(T) \cdot e^{\mu^{\bullet}}$,

$$
Z(T)=\int_{0}^{\infty} G(x) \cdot e^{-x} d x .
$$

Therefore, the neutrality equation (13) has the following form:

$$
Z(T) \cdot e^{\mu^{\bullet}}=\frac{N_{D}}{1+2 \exp \left(E_{D}^{*}+\mu^{*}\right)}-N_{A}
$$
$[4,5]$ :

This equation has the following analytical solution

$$
\mu^{\bullet}=\ln \left(\frac{n\left(N_{D}, N_{A}, n_{D}\right)}{Z(T)}\right) .
$$

The notation used in this formula are listed below:

$$
\begin{aligned}
n\left(N_{D}, N_{A}, n_{D}\right)= & \frac{n_{D}}{4}\left(\sqrt{\left(1+\frac{2 N_{A}}{n_{D}}\right)^{2}+\frac{8\left(N_{D}-N_{A}\right)}{n_{D}}}\right. \\
& \left.-\left(1+\frac{2 N_{A}}{n_{D}}\right)\right), \\
n_{D} & =Z(T) \exp \left(-\frac{E_{D}}{k T}\right) .
\end{aligned}
$$

The equation just listed is not adequate to describe degenerate current carriers.

The general analysis of equation (19) shows that the chemical potential $\mu^{\bullet}$ (20) determined from this equation has a maximum at some temperature $T_{e}$. The extremum $\mu_{e}^{\cdot}$ and a temperature value, where the reduced chemical potential has its maximum value, may be determined from the following equation system:

$$
\begin{gathered}
F\left(\mu^{\bullet}, T\right)=0, \\
\frac{d F\left(\mu^{\bullet}, T\right)}{d T}=0,
\end{gathered}
$$

where the function $F\left(\mu^{\bullet}, T\right)$ is given by the formula:

$$
F\left(\mu^{\bullet}, T\right)=Z(T) \cdot e^{\mu^{\bullet}}-\frac{N_{D}}{1+2 \exp \left(E_{D}^{\bullet}+\mu^{\bullet}\right)}+N_{A}=0 \text {. }
$$

The functional analysis of this system of transcendental equation shows that its solution always exists.

If the shielding parameter of a doped crystal $y \cong 2$, then by formula (15) the current carriers in this crystal are weak degenerate. This makes the solving of the neutrality equation (13) more complicated. The method of solving this problem is detaily described in work [5]. This solution is defined by complicated functions and they are a complex subject of analyses, then in this work this solution is not given.

The calculations of the thermal and kinetic properties of conducting crystals given in this work show us that all they are described by the following algorithmic functional:

$J\left(i, j, \mu^{\bullet}, T\right)=\int_{0}^{\infty} x^{i} u(x, T)^{j} G(x, T)\left(-\frac{d f_{0}\left(x, \mu^{\bullet}\right)}{d x}\right) d x,(21)$

with the indices $i=0,1,2 ; j=0,1,2$.

For calculations of the thermal crystal properties this functional has the such indices: $i=0 ; j=0$. Therefore, we have:

$$
\begin{aligned}
J\left(0,0, \mu^{\bullet}, T\right) & =\int_{0}^{\infty} G(x, T)\left(-\frac{d f_{0}\left(x, \mu^{\bullet}\right)}{d x}\right) d x \\
& =n\left(\mu^{\bullet}, T\right)=-\frac{1}{V}\left(\frac{d \Omega}{d \mu^{\bullet}}\right) .
\end{aligned}
$$

As it was shown above, the thermodynamic Gibbs potential

$\Omega=-2 V \int_{0}^{\infty} \frac{G(\varepsilon)}{\left(\exp \left(\frac{\varepsilon-\mu}{k T}\right)\right)} d \varepsilon=-2 V \int_{0}^{\infty} G(\varepsilon) f_{0}(\varepsilon, \mu) d \varepsilon$

fulfils this equation, and the general algorithmic 


$$
\begin{aligned}
& J\left(i, j, \mu{ }^{\bullet}, T\right)=\int_{0}^{\infty}\left(\frac{\varepsilon}{k T}\right)^{i} u(\varepsilon)^{j} G(\varepsilon)\left(-\frac{d f_{0}}{d \varepsilon}\right) d \varepsilon= \\
& =U(r, T)^{j} N_{C}(T) \int_{0}^{\infty} x^{i} \frac{E(x)^{(r j-j / 2+3 / 2)}}{\left(\frac{d E(x)}{d x}\right)^{2 j}}\left(-\frac{d f_{0}}{d x}\right) d x=U(r, T)^{j} N_{C}(T) I\left(i, j, \mu{ }^{\bullet}, T\right),
\end{aligned}
$$

$$
=U(r, T)^{j} N_{C}(T) \int_{0}^{\infty} x^{i} \frac{E(x)^{(r j-j / 2+3 / 2)}}{\left(\frac{d E(x)}{d x}\right)^{2 j}}\left(-\frac{d f_{0}}{d x}\right) d x
$$$$
=U(r, T)^{j} N_{C}(T) I\left(i, j, \mu^{\bullet}, T\right),
$$

where a dimensionless integral $I\left(i, j, \mu^{\bullet}, T\right)$ is given as:

$$
I\left(i, j, \mu^{\bullet}, \beta(T)\right)=\int_{0}^{\infty} x^{i} \frac{E(x)^{(r j-j / 2+3 / 2)}}{\left(\frac{d E(x)}{d x}\right)^{2 j}}\left(-\frac{d f_{0}}{d x}\right) d x .
$$

In crystals with a narrow energy gap $E_{G}$, the dispersion law (9) is described by the Kane's nonparabolic band:

$$
\frac{p^{2}}{2 m m_{n}}=\left(\varepsilon+\frac{\varepsilon^{2}}{E_{G}}\right)=\left(\varepsilon+\beta(T) \frac{\varepsilon^{2}}{k T}\right),
$$

where $\beta(T)=\frac{k T}{E_{G}}$ is the parameter of nonparabolicity. Hence, the dimensionless integral $I\left(i, j, \mu^{\bullet}, T\right)$ is given as:

$$
I(i, j, \mu, \beta(T))=\int_{0}^{\infty} x^{i} \frac{\left(x+\beta(T) x^{2}\right)^{(r j-j / 2+3 / 2)}}{(1+2 \beta(T) x)^{2 j}}\left(-\frac{d f_{0}}{d x}\right) d x .
$$

In crystals with a wide energy gap $E_{G}$, the nonparabolicity parameter $\beta(T)=\frac{k T}{E_{G}} \ll 1 \rightarrow 0$. That is to say in such crystals, the the dispersion law is a parabolic, and integral (24) takes the following form:

$$
I\left(i, j, \mu^{\bullet}, \beta(T)\right)_{\beta=0}=\left(\int_{0}^{\infty} x^{i} \frac{\left(x+\beta(T) x^{2}\right)^{(r j-j / 2+3 / 2)}}{(1+2 \beta(T) x)^{2 j}}\left(-\frac{d f_{0}}{d x}\right) d x\right)_{\beta=0}=F_{a(i, j)}\left(\mu^{\bullet}\right),
$$

where $a(i, j)=i+r j-j / 2+3 / 2$. In this formula, the function $F_{a(i, j)}\left(\mu^{\bullet}\right)$ is well-known the Fermi integral:

$$
F_{a(i, j)}\left(\mu^{\bullet}\right)=\int_{0}^{\infty} x a(i, j)\left(-\frac{d f_{0}\left(x, \mu^{\bullet}\right)}{d x}\right) d x .
$$

For crystals with non-degenerate current carriers, where their reduced chemical potential $\mu^{\bullet}<-4$, this integral is given as :

$$
F_{a(i, j)}\left(\mu^{\bullet}\right)=\Gamma(a(i, j)+1) e^{\mu^{\bullet}}
$$

where $\Gamma(a(i, j)+1)$ is the gamma function.

In a case of a strong degeneracy $\mu^{\bullet}>+4$, the Fermi integral is given as:

$$
F_{a(i, j)}\left(\mu^{\bullet}\right) \cong\left(\mu^{\bullet}\right)^{a(i, j)}
$$

Formulas (26), (27), (28) give the possibility to calculate the kinetic properties of crystal with a parabolic dispersion law for non-degenerate and strong degenerate current carriers.

In crystals with a narrow energy gap $E_{G}$, the nonparabolicity parameter, as a rule, fulfils the condition $\beta(T)=\frac{k T}{E_{G}}<1$. In this connecton we expand the integral $I\left(i, j, \mu^{\bullet}, \beta(T)\right)$ in a Taylor series of the parameter $\beta(T)$ and we restrict ourselves to the linear term of this expansion:

$$
I\left(i, j, \mu^{\bullet}, \beta(T)\right)=I\left(i, j, \mu^{\bullet}\right)\left(1+\frac{1}{I\left(i, j, \mu^{\bullet}\right)} \cdot\left(\frac{d I\left(i, j, \mu^{\bullet}, \beta(T)\right)}{d \beta(T)}\right)_{\beta(T)=0} \cdot \beta(T)\right)+\ldots=
$$




$$
=F_{a(i, j)}\left(\mu^{\bullet}\right)\left(1+(a(i, j)-i-4 j) \frac{F_{a(i, j)}\left(\mu^{\bullet}\right)}{F_{a(i, j)}\left(\mu^{\bullet}\right)} \cdot \beta(T)\right)=F_{a(i, j)}\left(\mu^{\bullet}\right)(1+\Delta(i, j, \mu \bullet) \cdot \beta(T))
$$

Since in the last formula $(a(i, j)-i-4 j) \frac{F_{a(i, j)}\left(\mu^{\bullet}\right)}{F_{a(i, j)}\left(\mu^{*}\right)}$. $\beta(T)=\Delta\left(i, j, \mu^{\bullet}\right) \cdot \beta(T)<1$, then it may be written in a form to be more conveniently to analysis:

$I\left(i, j, \mu^{\bullet}, \beta(T)\right) \cong F_{a(i, j)}\left(\mu^{\bullet}\right) \exp \left(\Delta\left(i, j, \mu^{\bullet}\right) \cdot \beta(T)\right)$.

Formula (29), together with the algorithmic functional (29), give us the possibility of analysis and calculate all set of the thermal and kinetic properties of the current carriers gas in conducting crystals with the Kane's dispersion law.

In crystals with a wide energy gap $E_{G}$, the nonparabolicity parameter $\beta(T)=\frac{k T}{E_{G}} \ll 1 \rightarrow 0$, as a rule, fulfils the condition $\beta(T)=\frac{k T}{E_{G}} \ll 1 \rightarrow 0$. Hence, we have:

$$
I\left(i, j, \mu^{\bullet}, \beta(T)\right) \cong F_{a(i, j)}\left(\mu^{\bullet}\right) \exp (0)=F_{a(i, j)}\left(\mu^{\bullet}\right) \text {. }
$$

Formula (29) implies that in isotropic crystals with a wide energy gap $E_{G}$, there is a parabolic energy dispersion law of current carriers and all set of the crystals thermal and kinetic properties is given by formula (30), that is by the appropriate Fermi integral.

In the cited work [5], it was shown that real semiconductor crystals have fundamental characteristics $\mu_{k}^{\cdot}\left(D i, m_{n}, T\right) \quad$ and $\quad n_{k}\left(D i, m_{n}, T\right) \quad$ and these characteristics depend on the crystal nature and do not depend on the concentration of dopant atoms. These characteristics can be determinated with the use of the following integral equations:

$$
\begin{aligned}
& \int_{0}^{\infty} g(x, T)\left(-\frac{d f_{0}\left(x, \mu_{k}^{\cdot}\right)}{d x}\right) d x=\frac{y 0^{2}}{4 D i}\left(\frac{m_{n}}{T}\right)^{1 / 2}, \\
& \int_{0}^{\infty} G(x, T)\left(-\frac{d f_{0}\left(x, \mu_{k}^{\cdot}\right)}{d x}\right) d x=n_{k}\left(D i, m_{n}, T\right) .
\end{aligned}
$$

For the Kane's dispersion law in this formula, the quantity $G(x, T)$ is given by $G(x, T)=\left(x+\beta(T) x^{2}\right)^{3 / 2}$, and $\quad g(x, T)=\frac{d G(x, T)}{d x}, \quad y 0=35.342, \quad \beta(T)=\frac{k T}{E_{G}}$; $f_{0}\left(x, \mu_{k}\right)$ is the known Fermi-Dirac function, $D i$ is the dielectric constant of the crystal.

Critical values of the reduced chemical potentials $\mu_{k}^{\bullet}\left(D i, m_{n}, T\right)$ and concentrations $n_{k}\left(D i, m_{n}, T\right)$ give the condition of turning the semiconductor crystal into metalic type of crystal. Therefore, if for a crystal the condition $\mu^{\bullet}<\mu_{k}^{\cdot}\left(D i, m_{n}, T\right), n\left(\mu^{\bullet}, T\right)<n_{k}\left(D i, m_{n}, T\right)$ holds, then this is a semiconductor type crystal, and if the condition $\mu^{\bullet}>\mu_{k}^{\cdot}\left(D i, m_{n}, T\right), n\left(\mu^{\bullet}, T\right)>n_{k}\left(D i, m_{n}, T\right)$ holds, this crystal has metallic properties.

In crystals of metals the current carriers concentration does not depend on temperature and the current carriers are strongly degenerate. In semiconductor crystals, they are weakly degenerate $\left(-4<\mu^{\bullet}<+1.2\right)$ or non-degenerate $\left(\mu^{\bullet}<-4\right)$, their concentration strongly dependent on temperature. Their chemical potential depends on temperature and it has a maximum in its temperature domain. The existence of this maximum shows that there are electron transitions from donor energy levels to the conduction band. Thus, the electron concentration grows as temperature increases. The impurity atoms concentrations and donors ionization energy may be determinated with the use of this extremum.

Budzhak Ya.S. - Professor, Doctor of Science;

Wactawski T. - Adjunct, Doctor of Philosophy in Physics.

[1] J.S. Budjak, Physics and Chemistry of Solid State 18(1), 7 (2017) (doi: 10.15330/pcss.18.1.7-14).

[2] Ya.S. Budzhak, T. Wacławski, Physics and Chemistry of Solid State 19(2), 134 (2018) (doi: 10.15330/pcss.19.2.134-138).

[3] Ya.S. Budzhak, T. Wacławski, Physics and Chemistry of Solid State 19(4), 303 (2018) (doi: 10.15330/pcss. 19.4.303-306).

[4] Ya.S. Budzhak, L.O. Vasilechko, Foundations of statistical theory of thermal and kinetic properties of semiconductor crystals (Liga-Pres, Lviv, 2016).

[5] Ya.S. Budzhak, A.O. Druzhinin, T. Wacławski, Modern Statistical Methods of Investigations of Properties of Crystals as Micro- and Nanoelectronics Materials (Publishing House of Lviv Polytechnic, Lviv, 2018). 


\title{
Я.С. Буджак ${ }^{1}$, Т. Вацлавський ${ }^{2}$
}

\section{До питання про проблеми статистичних розрахунків термодинамічних та кінетичних властивостей провідних кристалів}

\author{
${ }^{1}$ Національний університет «Львівська політехніка», м. Львів, Украӥна, e-mail: jabudjak@ ukr.net \\ ${ }^{2}$ Краківська політехніка, м. Краків, Польща, е-таil: tadeuszwaclawski00@gmail.com
}

В даній роботі описана елементарна модель кристалу та його термодинамічно рівноважний стан. Показано, що термодинамічні характеристики кристала в такому стані описуються великим термодинамічним потенціалом Гіббса. Якщо кристал виведений із стану термодинамічної рівноваги то в цьому стані він описується множиною кінетичних властивостей, які статистично розраховуються за допомогою великого термодинамічно нерівноважного потенціалу Гіббса. Термодинамічні і кінетичні властивості кристалів мають аналітичну залежність від закона дисперсії носіїв струму кристала та їх хімічного потенціалу. В роботі показано, що визначення закону дисперсії та хімічного потенціалу - це складні проблеми статистичної і кінетичної теорії властивостей кристалів.

Ключові слова: потенціал Гіббса, закон дисперсії, хімічний потенціал, дрейфова сила. 